\title{
Optical depths for gamma-rays in the radiation field of a star heated by an external X-ray source in LMXBs
}

\section{Application to Hercules X-1 and Scorpius X-1}

\author{
W. Bednarek and J. Pabich
}

\author{
Department of Astrophysics, University of Łódź, 90-236 Łódź, ul. Pomorska 149/153, Poland \\ e-mail: bednar@astro.phys.uni.lodz.pl
}

Received 1 October 2009 / Accepted 24 January 2010

ABSTRACT

\begin{abstract}
The surface of a low mass star inside a compact low mass X-ray binary system (LMXB) can be heated by an external X-ray source apparently caused by the accretion of mass onto a companion compact object (a neutron star or a black hole). As a result, the surface temperature of the star can become significantly higher than it is in the normal state resulting from thermonuclear burning. We investigate whether high energy electrons and gamma-rays, injected within the binary system, can efficiently interact with this enhanced radiation field. To decide this, we calculate the optical depths of the gamma-ray photons in the radiation field of such an irradiated star as a function of the phase of the binary system. Based on these calculations, we conclude that compact low mass X-ray binary systems may also become sources of high energy gamma-rays since conditions for the interaction of electrons and $\gamma$-rays are quite similar to those found within high mass TeV $\gamma$-ray binaries such as LS 5039 and LSI $303+61$. However, because of differences in the soft radiation field, the expected $\gamma$-ray light curves can differ significantly between low mass and high mass X-ray binaries. As an example, we apply these calculations to two well known LMXBs Her X-1 and Sco X-1. It is concluded that electrons accelerated to high energies inside these binaries should collide with a sufficient number of soft photon targets from the companion star to achieve efficient $\gamma$-ray production.
\end{abstract}

Key words. binaries: close - gamma rays: general - radiation mechanisms: non-thermal

\section{Introduction}

$\mathrm{TeV} \gamma$-rays have been observed from a few massive X-ray binaries (e.g., Aharonian et al. 2005a; Albert et al. 2006). Up to now, this $\gamma$-ray emission has not been reported from low mass X-ray binaries (see e.g., observations of SS 433, Aharonian et al. 2005b), which are very luminous X-ray sources $\left(L_{X} \sim\right.$ $10^{36-39} \mathrm{erg} \mathrm{s}^{-1}$, see the catalog of LMXB by Liu et al. 2007). However, LMXBs have not been monitored intensively by modern Cherenkov telescopes until now. In this paper, we attempt to demonstrate that very compact LMXBs are also promising targets for $\mathrm{TeV} \gamma$-ray astronomy.

LMXBs probably consist of a neutron star that accretes matter from a companion star. Their X-ray emission at times exhibits pulsations with the rotational period of the neutron star (Nagase 1989; Karino 2007). Some LMXBs also exhibit optical modulation with the period of the binary system, which can be interpreted as a result of external heating of the stellar surface by the X-ray source (e.g., Her X-1, Sco X-1).

The mechanism for particle acceleration and $\gamma$-ray production in the massive $\mathrm{TeV} \gamma$-ray binaries is not at present clear. Two general scenarios are considered. In the first, an energetic pulsar creates a relativistic wind that collides with the strong wind of the massive star creating a shock wave (e.g., Maraschi \& Treves 1984; Vestrand \& Eichler 1982; Harding \& Gaisser 1990; Tavani \& Arons 1997; Kirk et al. 1999). Electrons can be accelerated to $\mathrm{TeV}$ energies by the shock acceleration or in the not so well known mechanism occurring in the pulsar wind. In the second scenario, a compact object accretes matter from the massive star.
Electrons can be accelerated in the shock waves created either in the stream of matter expelled from the inner part of the accretion disk (a jet) (e.g. Levinson \& Blandford 1996; Georganopoulos et al. 2002; Romero et al. 2002) or at the border in which accreting matter is stopped by the rotating neutron star magnetosphere (Bednarek 2009a,b). The second scenario can also occur in the case of low mass X-ray binaries, hence the acceleration of particles to TeV energies should also occur within low mass X-ray binaries. We note that $\mathrm{TeV} \gamma$-ray emission is observed from a variety of cosmic sources (jets of active galaxies, vicinity of pulsars, supernova remnants, clusters of stars) that significantly differ in structure and physical parameters. Even if the parameters of objects responsible for the acceleration of particles within HMXBs and LMXBs differ significantly, this does not preclude acceleration of particles to TeV energies in LMXBs. However, the efficiency of the particle acceleration may differ. Searching for $\gamma$-ray emission from LMXBs with the present and future Cherenkov telescopes (e.g., CTA) will help us to answer this basic question. The aim of this paper is to ascertain the most favorable conditions for detection of $\mathrm{TeV} \gamma$-ray signal from the class of very compact LMXBs.

Efficient $\gamma$-ray production within massive binaries probably occurs due to the Comptonization by relativistic electrons of the soft radiation produced by the massive star. This strong radiation is not present inside the low mass binaries, where a normal star has a characteristic surface temperature of lower than $\sim 10^{4} \mathrm{~K}$. Therefore, efficient $\mathrm{TeV} \gamma$-ray production is in general not expected to occur there. However, in very compact LMXBs, a low mass star can be heated extensively by the X-ray source 
produced by the accretion of matter onto the companion compact object. As a result, some parts of the stellar surface can reach temperatures comparable to those observed in the case of high mass stars (i.e., a few $10^{4} \mathrm{~K}$ ). This soft radiation intensity is sufficient to generate relativistic electrons, which are accelerated inside these compact low mass binaries, and consequently $\mathrm{TeV} \gamma$-rays. In this paper, we calculate the optical depths of $\gamma$-ray photons in the radiation field of irradiated low mass star to check whether $\gamma$-ray production in these systems can occur efficiently and whether produced $\gamma$-rays can escape from the vicinity of the star. We note, that the cross sections for $\gamma$-ray production by means of inverse Compton scattering (ICS) and $\gamma$-ray absorption in collision with soft photons are comparable. Therefore, directions within the binary system in which significant absorption of $\gamma$-rays can occur should also correspond to the directions of efficient $\gamma$-ray production in the ICS process.

\section{Soft radiation from irradiated star}

The X-ray emission produced in the vicinity of a compact source in some close low mass $\mathrm{X}$-ray binaries can reach values of $\sim 10^{36-39} \mathrm{erg} \mathrm{s}^{-1}$. Part of this X-ray emission can illuminate the surface of a companion star. As a result, the surface temperature rises significantly. We calculate the temperature profile on the surface of the irradiated star by a point X-ray source located at the distance, $H$, from the center of the star. We assume that all $\mathrm{X}$-ray emission absorbed by the stellar surface is irradiated as black body emission of characteristic temperature $T(z)$, where $z$ is the distance measured from the direction defined by both the center of the star and the X-ray source, to a specific point on the stellar surface (see Fig. 1). Simple considerations infer the temperature profile on the stellar surface to be given by,

$$
\begin{aligned}
T(z) & =\left(T_{\star}^{4}+\frac{L_{\mathrm{X}} \cos \beta / 4 \pi \sigma_{\mathrm{SB}} R_{\odot}^{2}}{z^{2}+\left(H-\sqrt{R_{\star}^{2}-z^{2}}\right)^{2}}\right)^{1 / 4} \\
& \approx 7.3 \times 10^{4}\left(\frac{L_{38} \cos \beta}{z^{2}+\left(H-\sqrt{R_{\star}^{2}-z^{2}}\right)^{2}}\right)^{1 / 4} \mathrm{~K},
\end{aligned}
$$

where distances are defined in Fig. $1, L_{\mathrm{X}}=10^{38} L_{38} \mathrm{erg} \mathrm{s}^{-1}$ is the luminosity of the X-ray source, $\sigma_{\mathrm{SB}}$ is the Stefan-Boltzmann constant, $\beta=\pi-\mu-v, \cos \mu=z / R_{\star}, \tan v=\left(H-\sqrt{R_{\star}^{2}-z^{2}}\right) / z$, and $R_{\star}$ is the stellar radius. The distances $H, R, R_{\star}$, and $z$ are expressed in units of the Solar radius $R_{\odot}=7 \times 10^{10} \mathrm{~cm}$. The value of $z$ can change in the range from 0 to $z_{\max }=R_{\star} \sqrt{H^{2}-R_{\star}^{2}} / H$.

The distribution of temperatures on the surface of the irradiated star seen by the external observer located at different places outside the star is shown in Fig. 2. Two effects determine this soft radiation field at a specific place: (a) the distance, $R$, from the stellar surface determines the solid angle intercepted by the star; (b) the angle, $\alpha$, at which the hot region is seen (measured with respect to the direction defined by the X-ray source and the star). We note that for reasonable parameters of the low mass X-ray binary system (e.g., $L_{X}=10^{38} \mathrm{erg} \mathrm{s}^{-1}$, and $H=2 R_{\star}$ ), the surface temperature can increase, because of the irradiation of the star, to a few $10^{4} \mathrm{~K}$. This is comparable to the surface temperature of the massive stars within the high mass X-ray binaries. Therefore, we expect that the optical depths of $\mathrm{TeV} \gamma$-rays in the

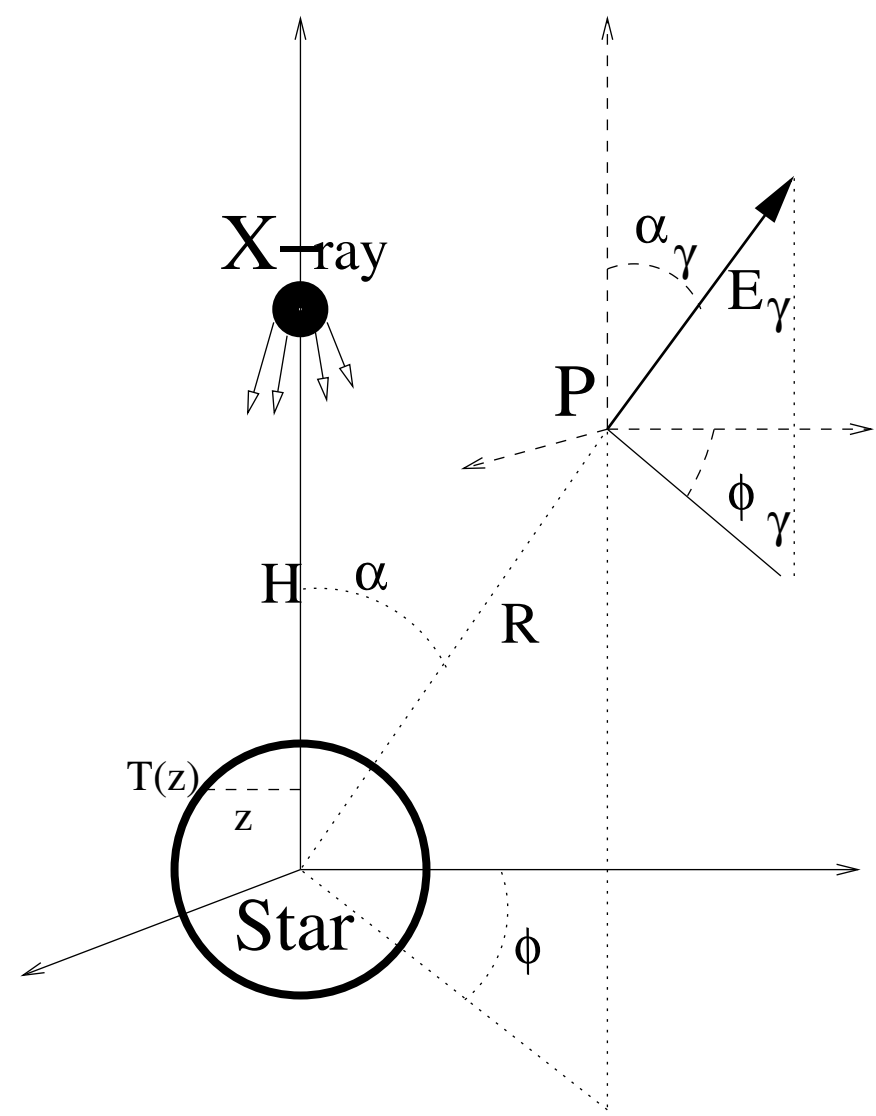

Fig. 1. Schematic picture of the geometrical situation considered in the paper. A $\gamma$-ray photon with energy $E_{\gamma}$ is injected from a place at the distance $R$ from the center of the star and a direction defined by the angle $\alpha$ and $\phi$. The $\gamma$-ray photon then propagates at the angles $\alpha_{\gamma}$ and $\phi_{\gamma}$. The star is irradiated by a point X-ray source (with the luminosity $\left.L_{X}\right)$ at the distance $H$. As a result of the irradiation, a temperature gradient appears on the stellar surface $(T(z))$, its maximum value occuring at the closest point to the X-ray source.

soft radiation of these irradiated low mass stars can also reach substantial values, exceeding unity in some cases.

Since the $\gamma$-ray photon injected at a specific place sees the hot part of the star at different angles, the soft radiation field seen by the $\gamma$-ray photon changes significantly during its propagation in the vicinity of the star (because of the change in both the viewing angle and the distance from the stellar surface). In Fig. 2, we show an example radiation field from the stellar surface seen by the $\gamma$-ray photon at different propagation distances $L$, assuming that the primary $\gamma$-ray is injected from the X-ray source. For the propagation angles $\alpha_{\gamma}>90^{\circ}$ (see Fig. 1), we note that the solid angle subtracted by the star at first increases since the $\gamma$-ray approaches the stellar surface. For small angles $\alpha_{\gamma}$, the solid angle subtracted by the star continuously drops with the $\gamma$-ray propagation path and the hot region becomes less visible.

\section{The optical depths of gamma-rays}

In the case of fixed surface temperature, the optical depths of $\gamma$-ray photons injected at an arbitrary distance from the surface of the star were calculated for the first time in the general case by Bednarek $(1997,2000)$. With respect to previous calculations (e.g., Moskalenko et al. 1993), Bednarek also took into account the dimensions of the star, which allowed them to apply their 

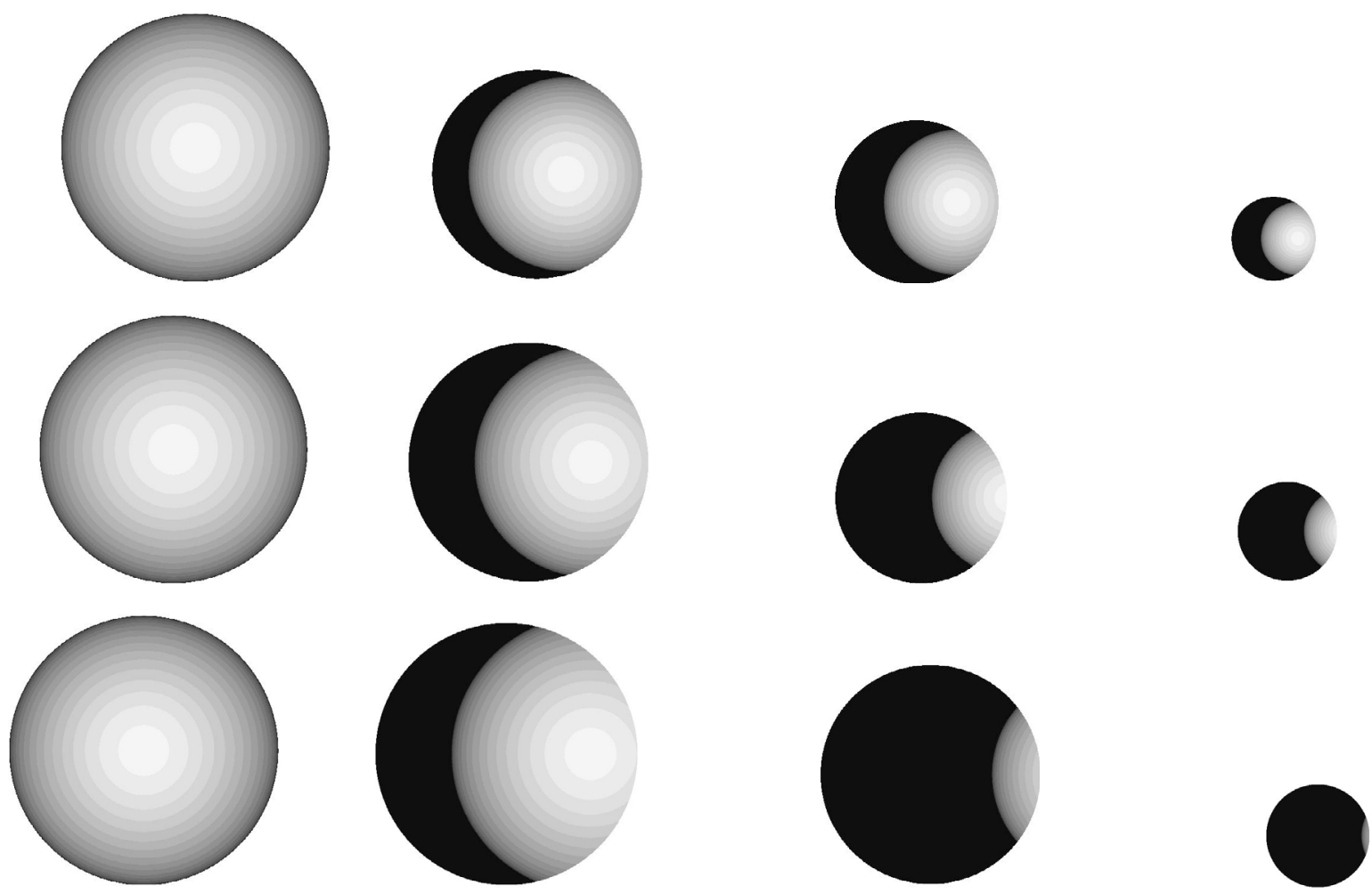

Fig. 2. The radiation field seen by a $\gamma$-ray photon at its different locations along the direction of propagation $L$. This radiation is due to the irradiation by an external X-ray source of luminosity $10^{38} \mathrm{erg} \mathrm{s}^{-1}$ located at the distance $H=2 R_{\star}$ from the center of the star. $\gamma$-rays are also injected at the distance $H=2 R_{\star}$ but at different angles $\alpha_{\gamma}=45^{\circ}$ (upper panel), $90^{\circ}$ (middle panel), and $120^{\circ}$ (bottom panel) with respect to the line defined by the injection point and the center of the star. The propagation distance is equal to $L=0$ (left), $R_{\star}$ (left-middle), $3 R_{\star}$ (right-middle), and $5 R_{\star}(r i g h t)$. The star has the radius $R_{\star}=R_{\odot}$ equal to the radius of the Sun and the surface temperature $T_{\star}=6000 \mathrm{~K}$. The greyscale denotes the range of temperatures starting from $7.5 \times 10^{4} \mathrm{~K}$ (white) to $6 \times 10^{3} \mathrm{~K}$ (black).

method to very compact binaries such as e.g., Cyg X-3. These calculations are necessary for IC $e^{ \pm}$pair cascades developing within the massive binaries since in principle secondary $e^{ \pm}$pairs and $\gamma$-rays can appear everywhere within the binary. They can also fall onto the surface of the companion star. The optical depths of $\gamma$-rays calculated in the radiation field of the star with specific parameters can be easily rescaled to the cases of massive stars with other surface temperatures and radii (Bednarek 2009a). For example, $\gamma$-ray photons with energies, $E_{\gamma}^{\mathrm{o}}$, propagating from a specific distance $D$ from the star, and in a direction (defined by the angle $\alpha$ ) approaching close to the star with specific parameters $\left(T_{\mathrm{o}}\right.$ and $R_{\mathrm{o}}$ ) are related to the optical depths around arbitrary stars with $T_{\star}$ and $R_{\star}$ by,

$\tau\left(\frac{E_{\gamma}^{\mathrm{o}}}{S_{\mathrm{T}}}, T_{\star}, R_{\star}, D, \alpha\right)=S_{T}^{3} S_{R} \tau\left(E_{\gamma}^{\mathrm{o}}, T_{\mathrm{o}}, R_{\mathrm{o}}, D, \alpha\right)$,

where $S_{T}=T_{\star} / T_{\mathrm{o}}, S_{R}=R_{\star} / R_{\mathrm{o}}$, and the distance $D$ is measured in stellar radii. In the context of massive stars inside the high mass X-ray binaries detected in the $\mathrm{TeV} \gamma$-rays these $\gamma$-ray optical depths have been frequently discussed (see e.g., Böttcher \& Dermer 2005; Bednarek 2006; Dubus 2006).

Here we are interested in a more complicated scenario appropriate to low mass X-ray binary systems. In LMXBs, the companion stars produce relatively weak soft radiation field due to its low surface temperature resulting from nuclear burning. However, a nearby strong X-ray source can significantly increase the surface temperature of a star because of the irradiation process (as discussed in Sect. 2). Therefore, relativistic electrons injected not far from the surface of the low mass star in a compact
X-ray binary can also suffer strong energy losses because of the ICS process. Primary $\gamma$-rays and secondary cascade $\gamma$-rays can be efficiently absorbed in this soft radiation field. To check whether these processes may become important, we calculate the optical depths of $\gamma$-rays in this more complicated scenario following the standard prescription

$$
\tau=\int_{\ell} \mathrm{d} l \int \mathrm{d} \epsilon \mathrm{d} \Omega n(l, \epsilon, \Omega) \sigma_{\gamma \gamma}(\epsilon, \theta)(1-\cos \theta),
$$

where $n(l, \epsilon, \Omega)$ is the differential density of soft photons with energy $\epsilon$ that arrive from the low mass star inside the solid angle $\Omega$ to the instantaneous location of the $\gamma$-ray photon at the propagation distance $l, \sigma_{\gamma \gamma}$ is the $e^{ \pm}$pair production cross-section, $\theta$ is the angle between the momentum vectors of the gamma-ray and soft photon, and $\ell$ denotes the path along the propagation direction of the gamma-ray photon in the soft radiation field.

We investigate the optical depths of $\gamma$-rays as a function of their energies and other free parameters describing the geometry of the picture, such as the injection distance $R$ and the angle $\alpha$ (see Fig. 1). The low mass stars with different radii and surface temperatures are considered. For the purpose of the example calculations, the X-ray luminosity of the compact object (a neutron star) is fixed on $L_{X}=10^{38} \mathrm{erg} \mathrm{s}^{-1}$. The optical depths as a function of $\gamma$-ray photon energy for selected injection angles and distances from the companion star are shown in Fig. 3. The calculations show that the optical depths are significant for some range of investigated energies of $\gamma$-ray photons. They are clearly above unity provided that $\gamma$-rays are injected within $R \sim 2 R_{\star}$ from the center of the star at a part of the hemisphere containing 

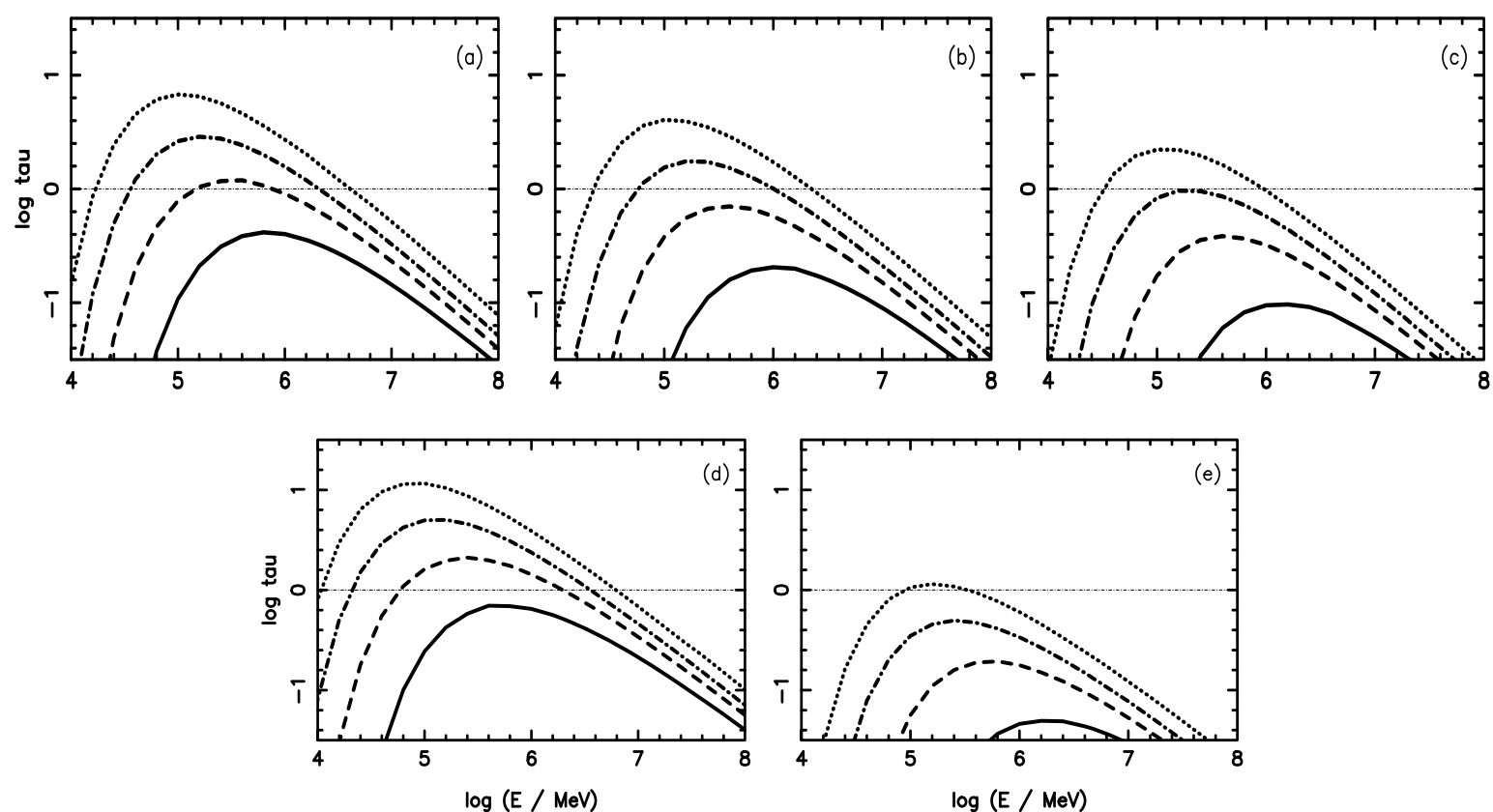

Fig. 3. The optical depths for $\gamma$-rays in the radiation field created by a low mass star irradiated by an external X-ray source. It is assumed that the $\mathrm{X}$-ray source is at the distance $H=2 R_{\star}$ (upper panel) from the center of the star. The $\gamma$-rays are injected at different distances from the star (see Fig. 1): $R=1.5 R_{\star}$ (left figure), $2 R_{\star}$ (middle), and $3 R_{\star}$ (right), and the angles $\alpha=0^{\circ}$ and $\phi=0^{\circ}$. The $\gamma$-ray photon moves at different angles $\alpha_{\gamma}=30^{\circ}$ (solid), $60^{\circ}$ (dashed), $90^{\circ}$ (dot-dashed), and $120^{\circ}$ (dotted). The optical depths for other distances of the X-ray source and injection places of $\gamma$-rays, $H=1.5 R_{\star}$ and $R=1.5 R_{\star}$ and $H=3 R_{\star}$ and $R=3 R_{\star}$, are shown in the bottom panel of the left and right figures, respectively. The parameters of the star in the binary system are: $T_{\star}=6000 \mathrm{~K}$, and $R_{\star}=R_{\odot}=7 \times 10^{10} \mathrm{~cm}$.
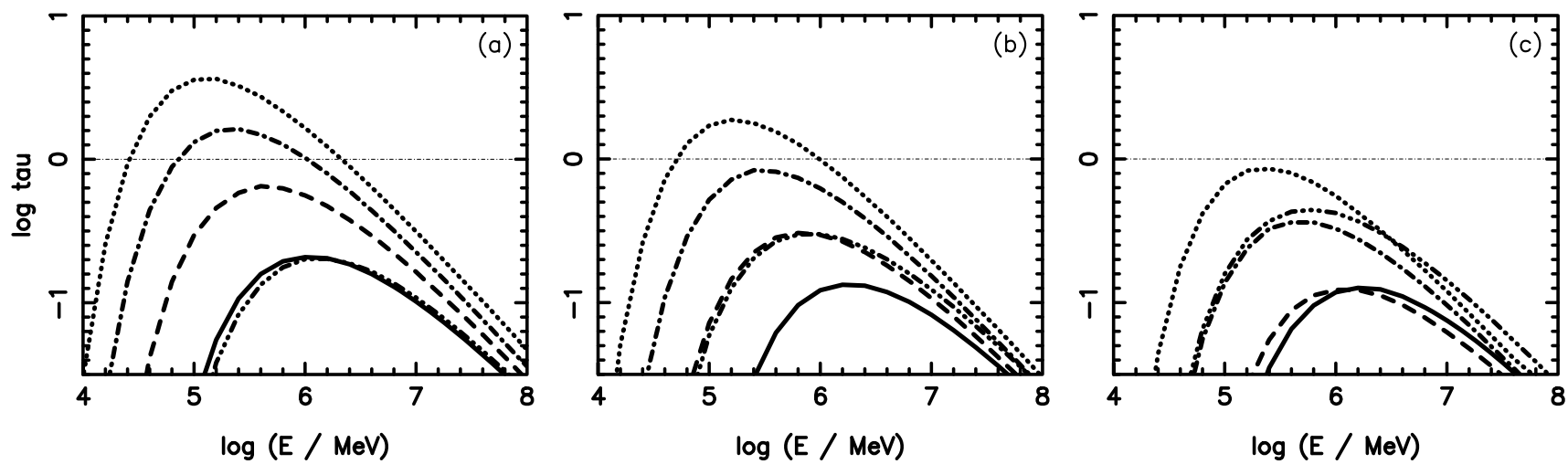

Fig. 4. As in Fig. 3 but for the X-ray source at the distance $H=2 R_{\star}$, injection distance of $\gamma$-rays from the star $R=1.5 R_{\star}$ and different angles of the injection place $\alpha=10^{\circ}$ (left figure), $20^{\circ}$ (middle), and $30^{\circ}$ (right). The $\gamma$-ray photon moves at different angle $\alpha_{\gamma}=1^{\circ}$ (triple-dot-dashed curve), $31^{\circ}$ (solid), $61^{\circ}$ (dashed), $91^{\circ}$ (dot-dashed), and $121^{\circ}$ (dotted).

the star. We note that the optical depths increase also for stars with lower radii in the case of similar compactness of the binary system (expressed as the ratio of the injection distance to the radius of the star). This is caused by the fact that irradiating X-ray source being closer to the stellar surface in these cases. We note that the amount of power supplied to the stellar surface from the $\mathrm{X}$-ray source increases as a square of its distance but the optical depths depend only linearly on this distance. Owing to the closer $\mathrm{X}$-ray source, the surface is heated to higher temperature.

In Fig. 4, we show how the optical depths depend on the angle $\alpha$, which is the angle between the direction defined by both the location of the X-ray source and by the center of the star and the injection place of $\gamma$-rays and the center of the star (see Fig. 1). This situation may correspond to the case of injection of $\gamma$-rays from different places of the jet launched from the compact object. We note, that for larger angles $\alpha$ the hottest region on the stellar surface is also seen at larger angles. Therefore, the effective radiation field from the stellar surface seen from the injection place of the $\gamma$-ray drops significantly. As a consequence, the optical depths for $\gamma$-ray photons are lower (compare e.g., Figs. 4a,c).

In summary, we conclude that the optical depths of $\gamma$-rays can also exceed unity in very compact low mass X-ray binary systems. Therefore, we predict that these LMXBs can become sources of $\mathrm{GeV}-\mathrm{TeV} \gamma$-rays as high mass X-ray binaries are, provided that the acceleration mechanism of electrons to $\mathrm{TeV}$ energies still operates.

\section{Gamma-ray optical depth light curves}

We also investigate how the $\gamma$-ray optical depths can change in the case of a low mass compact binary system in which the X-ray 


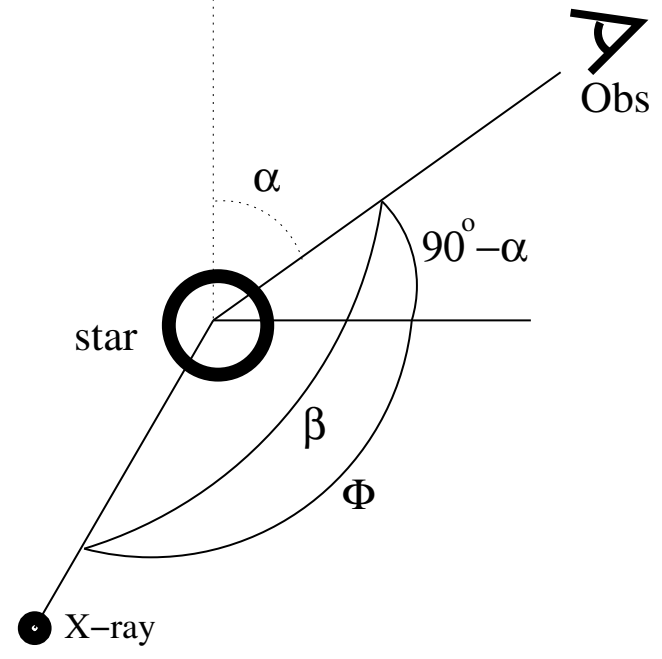

Fig. 5. The geometrical situation considered in the case of a compact $\mathrm{X}$-ray source on an orbit around a low mass star. The X-ray source irradiates the stellar surface heating it to a temperature significantly above that one caused by nuclear burning. The observer sees the heated stellar surface at the angle $\beta$ measured with respect to the direction defined by the X-ray source and the star. The system is inclined at the angle $\alpha$ in respect to the observer. The X-ray source and the $\gamma$-ray source are located at a phase $\Phi$ with respect to the plane of the observer.

source is on a circular orbit around the solar-type star. The angle $\beta$ between the direction towards the observer and the direction defined by the X-ray source and the star can change significantly depending on the inclination angle $\alpha$ of the binary system. In this case, $\beta$ is determined by the phase $\Phi$ of the X-ray source (for the geometrical situation see Fig. 5).

The example $\gamma$-ray optical depth light curves are calculated for the range of the inclination angles of the binary system and specific energies of $\gamma$-ray photons injected in the direction of the observer. The results for the X-ray source on a circular orbit of radius $R_{\mathrm{b}}=2 R_{\star}$ and the injection place of $\gamma$-rays also at the $\mathrm{X}$-ray source are shown in Fig. 6 . We note the characteristic optical depth light curves in the case of the non-eclipsing systems with inclination angles that differ from zero. In this case, the optical depths reach the maximum when the X-ray source (and also $\gamma$-ray source) is behind the star (with respect to the observer). At the peak of the maximum, the optical depths are larger for larger inclination angles of the binary system. For the considered parameters of the binary system, the optical depths are above unity at least for some range of phases close to the phase $\Phi=0.5$. In the case of the eclipsing binaries, the observer should see two peaks in the optical depth light curve due to the eclipse of the $\mathrm{X}$-ray and $\gamma$-ray source by the star. At the phases at which the $\gamma$-ray optical depth light curves reach the maximum, we note that the hot, irradiated part of the stellar surface is not clearly visible. Therefore, it is natural to expect an anticorrelation between the maximum of the $\gamma$-ray light curve and the maximum of the optical light curve from these low mass binary systems. This prediction might be useful in increasing the probability of the detecting the GeV-TeV $\gamma$-ray signal from compact X-ray luminous LMXBs.

Since the assumed orbit of the X-ray source (and $\gamma$-ray source) is circular, the light curve looks symmetric. This light curve can change significantly when the distance of the X-ray source varies with the phase of the binary. To obtain an

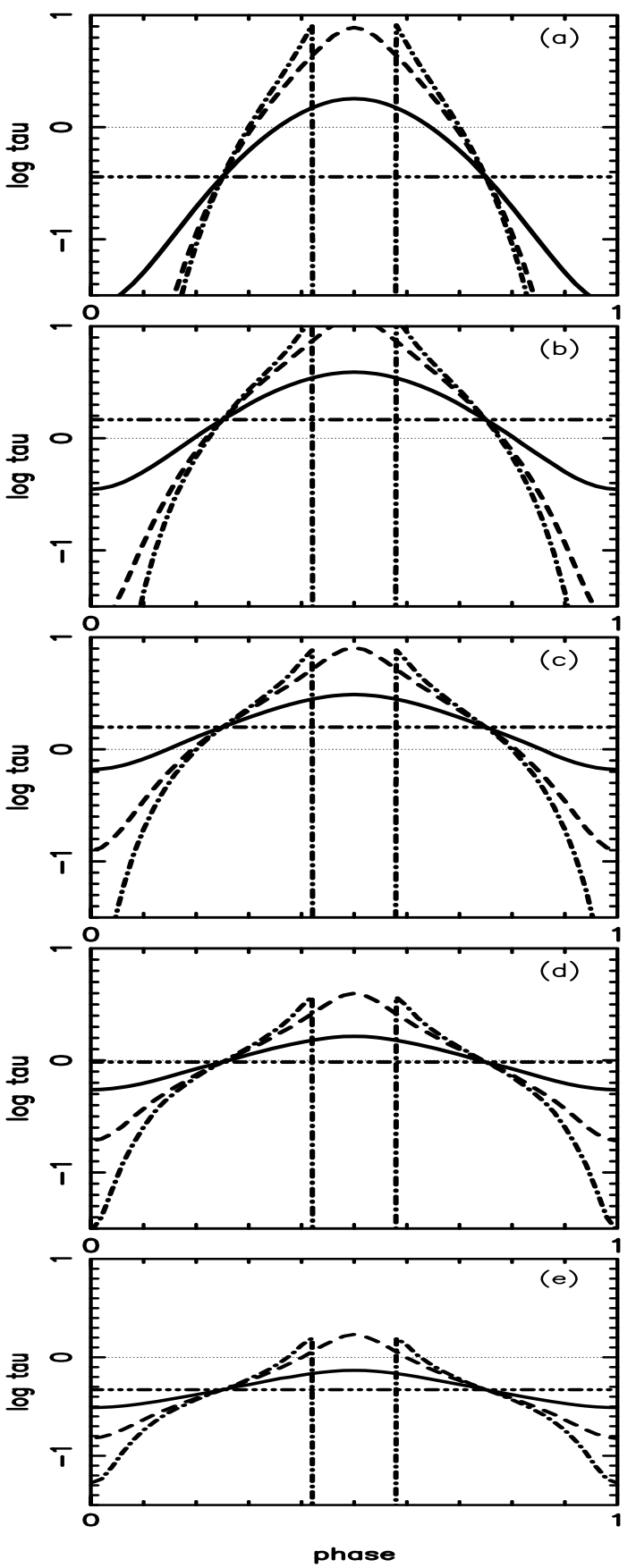

Fig. 6. The dependence of the $\gamma$-ray optical depths in the thermal radiation of the irradiated star as a function of the phase of the binary system. The phase is measured from the situation in which the X-ray source is in front of the star (in respect to the observer). Specific figures show the $\gamma$-ray optical depth light curves for selected energies of $\gamma$-ray photons equal to: $33 \mathrm{GeV}$ a), $100 \mathrm{GeV}$ b), $330 \mathrm{GeV}$ c), $1 \mathrm{TeV}$ d), and $3.3 \mathrm{TeV}$ e). The light curves for different inclination angles of the binary system are shown for: $\alpha=0^{\circ}$ (triple-dot dashed curve), $30^{\circ}$ (solid), $45^{\circ}$ (dashed), $60^{\circ}$ (dashed), and $89^{\circ}$ (dot-dashed). The thin dotted line indicates the optical depth equal to unity. The parameters of the star are $R_{\star}=R_{\odot}$ and its surface temperature $T_{\star}=6000 \mathrm{~K}$. The orbit of the X-ray source is circular with the radius $R_{\mathrm{b}}=2 R_{\star}$. The $\gamma$-rays are injected from the vicinity of the $\mathrm{X}$-ray source.

impression of the influence of the ellipticity of the orbit, we calculate the optical depth light curves for other distances of the $\mathrm{X}$-ray and $\gamma$-ray sources from the star. In Fig. 7, we investigate 
the optical depths for the orbits of the $\mathrm{X}$-ray source in the range $R_{\mathrm{b}}=2-3 R_{\star}$ and the distances of the $\gamma$-ray source in the range $R=1.5-3 R_{\star}$. We note a significant dependence of the optical depths on the two parameters $R_{\mathrm{b}}$ and $R$. However, for the complete range of these parameters, the optical depths are always above unity at some phases. Therefore, we expect that intensive absorption (and also production) of $\gamma$-ray photons might occur in the case of compact low mass X-ray binary systems. However in the realistic case, the spectrum of $\gamma$-ray photons should be generated by a the complicated cascade process triggered by a highly anisotropic soft radiation from this irradiated star. This cascade scenario will be clearly more complicated than considered up to now for the $\gamma$-ray production inside the massive binary systems (see e.g., early results by Bednarek 2000).

For comparison, we also show the $\gamma$-ray light curves for the case of a stellar mass companion in the giant phase (other parameters of the binary as in Fig. 7b). Because of the greater distance to the X-ray source from the surface, the optical depths are clearly lower than in the case of a binary system with a solar mass star on the main sequence. However, for a small range of phases and relatively large inclination angles of the binary system, the optical depths overcome unity. Therefore, in some geometrical situations these more geometrically extended binaries (i.e., with longer orbital periods) can provide conditions for efficient production of $\mathrm{GeV}-\mathrm{TeV} \gamma$-rays.

Calculated here $\gamma$-ray optical depth light curves allow us to conclude that the highest fluxes of $\mathrm{GeV} \gamma$-rays should be expected in the phases where the optical depths are clearly above unity. In these cases, the cascading effects degrade part of the primary $\gamma$-ray spectrum in the $\mathrm{TeV}$ energy range by means of efficient cascading. On the other hand, the highest fluxes of $\mathrm{TeV}$ $\gamma$-rays are expected in phases where the optical depths are close to unity. In this case, primary $\mathrm{TeV} \gamma$-rays are efficiently produced and not severely absorbed in the IC $e^{ \pm}$pair cascades. Based on the analysis of Figs. 6 and 7, we expect the highest fluxes of the $\mathrm{GeV} \gamma$-rays when the X-ray source (a compact object) is behind the normal star (except the eclipsing binaries). The highest fluxes of the $\mathrm{TeV} \gamma$-rays are expected during the intermediate phases, i.e., just before and after the highest fluxes of $\mathrm{GeV} \gamma$-rays.

\section{The example cases of LMXBs}

As an example, we show the $\gamma$-ray optical depth light curves for two LMXB systems that exhibit optical modulation with the binary system period. This modulation is interpreted as being caused by the irradiation of the companion star by the X-ray source (e.g., Milgrom 1976). They have been previously reported as possible $\mathrm{TeV}-\mathrm{PeV} \gamma$-ray sources.

\section{1. $\operatorname{Her} X-1$}

The famous LMXB, Her X-1, is characterised by the X-ray luminosity $L_{\mathrm{x}}=6 \times 10^{37} \mathrm{erg} \mathrm{s}^{-1}$ (White et al. 1983). The companion star has the radius $R_{\star}=3.86 R_{\odot}$ and mass $M_{\star}=2 M_{\odot}$ and a neutron star in orbit has a radius $R_{\mathrm{b}}=8.61 R_{\odot}$. The inclination of the binary system was estimated to be $\alpha=80_{-5}^{+8}$ degrees and its orbital period is 1.7 days (Nagase 1989).

Her X-1 was claimed to be a pulsed $\mathrm{TeV} \gamma$-ray source by the Whipple Collaboration (e.g. Gorham et al. 1986; Lamb et al. 1998). However, subsequent analysis did not confirm these reports (Reynolds et al. 1991). The modern Cherenkov telescope collaborations have not reported any additional observations of this source.
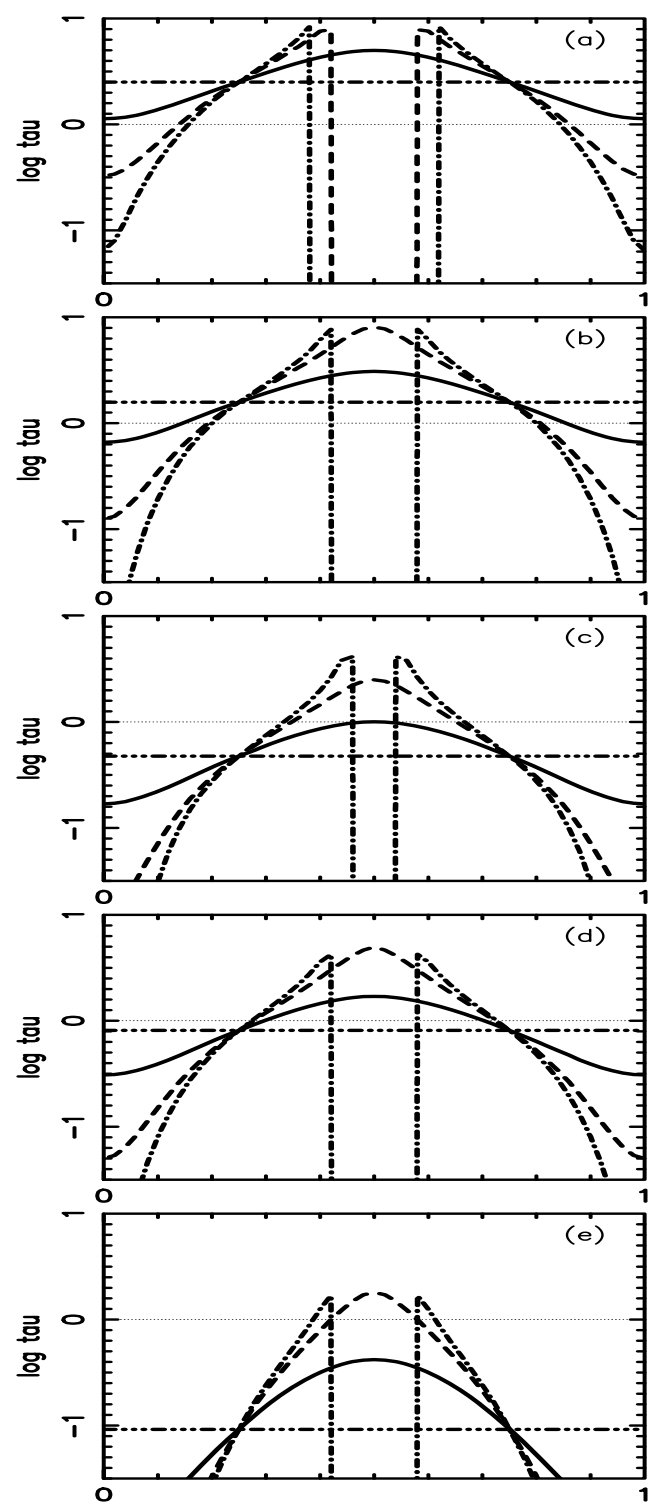

Fig. 7. As in Fig. 6 but for different distances of the X-ray source from the star $\left(R_{\mathrm{b}}\right)$ and different injection places of $\gamma$-ray photons $(R): R_{\mathrm{b}}=$ $2 R_{\star}$ and $\left.R=1.5 R_{\star} \mathbf{a}\right), R_{\mathrm{b}}=2 R_{\star}$ and $\left.R=2 R_{\star} \mathbf{b}\right), R_{\mathrm{b}}=3 R_{\star}$ and $R=3 R_{\star} \mathrm{c}$ ), and $R_{\mathrm{b}}=3 R_{\star}$ and $\left.R=2 R_{\star} \mathrm{d}\right)$. The case of the solar mass star in the giant phase is considered $\left(R_{\star}=100 R_{\odot}\right.$ and $\left.T_{\star}=3000 \mathrm{~K}\right)$ for $R_{\mathrm{b}}=2 R_{\star}$ and $R=2 R_{\star}$ (see $\left.\mathrm{e}\right)$ ). It is assumed that the location of $\gamma$-ray injection is always on the line defined by the X-ray source and the star. The energies of $\gamma$-ray photons were fixed to be $330 \mathrm{GeV}$.

We calculated the $\gamma$-ray optical depth light curves for the parameters of Her X-1. We assumed that the source of primary $\gamma$-rays (produced by accelerated electrons) is close to the X-ray source. The results are shown in Fig. 8 for selected $\gamma$-ray photon energies. It is clear that the optical depths are greater than unity for some relatively small range of phases in the case of $\gamma$-rays with energies in the range $E_{\gamma}=0.1-1 \mathrm{TeV}$. The optical depths can reach the maximum values $\tau \sim 3$. Therefore, the possible cascading effects have to be taken into account when calculating detailed $\gamma$-ray spectrum escaping towards the observer. However, the primary $\gamma$-ray spectrum should not be very severally degraded at the $\mathrm{TeV} \gamma$-ray energies as expected in the case of massive binary systems, such as LS 5039 or LSI 61 303. We predict that the highest fluxes of GeV-TeV $\gamma$-rays should appear in 
the range of phases just before and after the eclipse of the X-ray source by the companion star, i.e., in the ranges $\Phi \sim 0.35-0.45$ and $0.55-0.65$. We note that zero phase corresponds to the situation where the X-ray compact source is in front of the companion star. This $\gamma$-ray emission should be unticorrelated with the optical emission from the part of the stellar surface irradiated by the X-ray source. Evidence of the optical modulation with the period of the binary system Her X-1 has been clearly detected.

For the injection place of primary $\gamma$-rays which significantly differ from the location of the X-ray source (e.g. farther from the $\mathrm{X}$-ray source along the jet or at the shock structure), the phase patterns of the $\gamma$-ray optical depths should of course change accordingly.

\section{2. $S \operatorname{co} X-1$}

The second binary system, Sco X-1, has significantly different parameters from Her X-1. It contains a low mass star, $M_{\star}=$ $0.5 M_{\odot}$, and a neutron star (Liu et al. 2007). Sco X-1 belongs to the class of $\mathrm{Z}$ sources with three states of $\mathrm{X}$-ray emission characterised by the luminosities $L_{\mathrm{x}}=(4-12) \times 10^{37} \mathrm{erg} \mathrm{s}^{-1}$ (Hasinger $\&$ van der Klis 1989). The accretion disk around the neutron star in this binary system launches a jet. Therefore, the object also belongs to the class of microquasars. The inclination angle of the binary system was estimated to be $\alpha=44^{\circ} \pm 6^{\circ}$ (Fomalont et al. 2001). The period of the binary system is $18.9 \mathrm{~h}$ (Gottlieb et al. 1975). In our calculations, we apply the radius of the companion star $R_{\star}=1 R_{\odot}$ (evolved subgiant, Steeghys \& Casares 2002), the radius of the binary system $R_{\mathrm{b}}=2.14 r_{\odot}=1.5 \times 10^{11} \mathrm{~cm}$, the inclination of the binary system $\alpha=44^{\circ}$, and the power of the X-ray source $10^{38} \mathrm{erg} \mathrm{s}^{-1}$. Sco X-1 has been also claimed as a TeV-PeV $\gamma$-rays source but with low significance (e.g., Brazier et al. 1990; Tonwar et al. 199). Also these results have not been confirmed by latter observations.

As for Her X-1, the $\gamma$-ray optical light curves were calculated as a function of the energy of $\gamma$-ray photons. We note that Sco X-1 is more compact binary system than Her X-1. Therefore, the range of phases, for which the $\gamma$-ray optical depths are above unity, is significantly broader. The absolute values of the optical depths are also larger. We therefore expect much stronger modification of the escaping $\gamma$-ray spectra by IC $e^{ \pm}$pair cascading effects. The TeV $\gamma$-ray emission can then be severally attenuated for the phases close to $\sim 0.5$ and the $\mathrm{TeV} \gamma$-ray light curve may show two minima, the first due to inefficient production in the region close to $\sim 0.0$ phase and the second due to strong absorption in the region close to the phase $\sim 0.5$. In contrast, the $\mathrm{GeV} \gamma$-ray light curve should exhibit one broad maximum centered on the phase 0.5 . These conclusions are derived by assuming the circular orbit of the compact object around the companion star. A more detailed analysis is at present not possible because of the lack of information about the orbit of the compact object in Sco X-1 (i.e., ellipticity, phase of periastron).

It is not clear at present whether the companion star in Sco X-1 is really efficiently irradiated by the X-ray source. Early calculations by Milgrom (1976) suggest that irradiation is important and consistent with observational results at that time. In these calculations, relatively small inclination angles were considered (up to $i \sim 6^{\circ}$ ). The analysis of more recent observations infers more intermediate inclination angles (Fomalont et al. 2001; Steeghys \& Casares 2002), which were applied in this paper. The present lack of strong modulation of UV emission might be understood by assuming that a significant part of the hot region on the stellar surface is shadowed by the large accretion

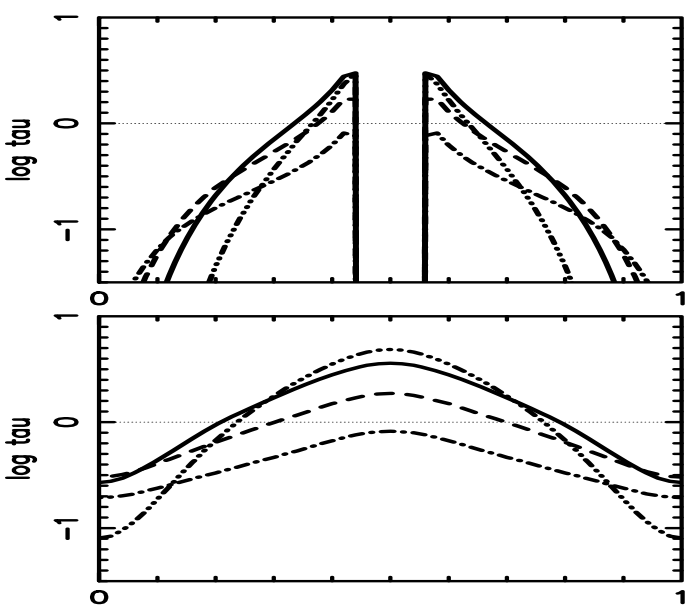

Fig. 8. The $\gamma$-ray optical depth light curves for two binary systems with the parameters mentioned in the main text: Her X-1 (upper figure) and Sco X-1 (bottom). Specific curves show results for different energies of $\gamma$-ray photons: $100 \mathrm{GeV}$ (triple-dot-dashed curve), $330 \mathrm{GeV}$ (solid), $1 \mathrm{TeV}$ (dashed), and $3.3 \mathrm{TeV}$ (dot-dashed). It is assumed that the injection place of the $\gamma$-rays is at the location of the X-ray source.

disk in this system. We note that the outer radius of this accretion disk was estimated on $\sim 6 \times 10^{10} \mathrm{~cm}$ (Vrtilek et al. 1991), which is comparable to the separation of stars in this binary.

\section{Discussion and conclusions}

We have demonstrated that the optical depths for $\mathrm{TeV} \gamma$-rays, which have been injected inside the LMXBs close to the surface of the companion stars irradiated by the X-ray sources, are larger than unity for specific locations of the observer. It is then straighforward to show that because of similar cross-section for the $\gamma-\gamma \rightarrow e^{ \pm}$absorption process and the cross-section for the ICS process of soft photons by relativistic electrons, the optical depths for electrons on these same stellar radiation should have similar values. Therefore, it is expected that the production and cascading of $\gamma$-rays can be efficient in the LMXBs in which the companion star is effectively heated by the close $\mathrm{X}$-ray source. However, the optical depths of irradiated low mass stars are generally lower than the optical depths expected in the HMXBs detected in the TeV $\gamma$-rays (such as LS 5039 or LSI $303+61$; e.g., Bednarek 2006). The cascading effects in the LMXBs should then not occur so efficiently as shown for those sources (Bednarek 2006b, 2007; Sierpowska-Bartosik \& Torres 2009, 2009). Owing to these substantial optical depths, we expect that the GeV-TeV $\gamma$-ray emission can also be efficiently produced within compact LMXBs in which the compact X-ray source strongly irradiates the companion star provided that electrons are accelerated to $\mathrm{TeV}$ energies also inside LMXBs. The mechanism of particle acceleration inside LMXBs can either differ from one operating within the HMXBs or can be quite similar. The $\mathrm{TeV} \gamma$-rays are observed from different types of sources (e.g., jets of AGNs, vicinity of pulsars, or shocks of supernova remnants). Therefore, different conditions around compact objects in HMXBs and LMXBs can accelerate particles to $\mathrm{TeV}$ energies. On the other hand, $\mathrm{TeV} \gamma$-ray emission inside LMXBs may be produced in scenarios similar to those proposed for HMXBs (e.g., accreting neutron star scenario as proposed by Bednarek 2009b). However, as we have shown, the $\mathrm{TeV} \gamma$-ray emission from LMXBs should be limited to specific narrow range of phases that are expected to differ from the range 
of phases observed in the HMXBs due to differences in the soft radiation field (see the considered example cases of Her X-1 and Sco X-1).

Based on the results of calculations of the optical depths and a simple application of the law of gravity, we can envisage that LMXBs are expected to be potential GeV-TeV $\gamma$-ray sources. We conclude that in the case of the solar type star in the evolutionary phase on the main sequence $\left(R_{\star}=R_{\odot}\right.$ and $\left.M_{\star}=M_{\odot}\right)$ and the orbit of radius $R_{\mathrm{b}}<3 R_{\star}$, the $\gamma$-rays may efficiently absorb, which means also their efficient production in IC process by electrons. This will occur provided that the period of the binary system is shorter than $P_{\mathrm{b}} \sim 17 R_{\star}^{3 / 2} M_{\star}^{-1 / 2} \mathrm{~h}$, where $R_{\star}$ and $M_{\star}$ are in the solar units. We note that many LMXBs have orbital periods within this limit (Liu et al. 2007).

Based on the analysis of the optical depth light curves, we conclude that the emission patterns of escaping $\gamma$-rays from the low mass binary system depend strongly on its inclination angle. We predict that in the simplest considered model of a point-like source of primary $\gamma$-rays on a circular orbit around irradiated low mass star, the observer located at:

- Small inclination angles detects significant GeV-TeV $\gamma$-ray emission only from very compact binaries in which the compact object is at the distance of less than 2 stellar radii from the companion star (compare the triple-dot dashed lines with the thin dotted lines in Figs. 6 and 7). In this case, the modulation of the UV emission from an irradiated companion star with the period of the binary system may be significantly reduced since the hottest parts of the stellar surface are not well visible.

- Intermediate inclination angles detects a single pulse of $\gamma$-ray emission with the maximum corresponding to the phases when the X-ray source is behind the companion star. In this case, the observer should detect significant modulation of the UV emission from the stellar surface, which in addition should be modulated with the period of the binary system.

- Large inclination angles and eclipsing binaries detects two narrow pulses of $\gamma$-rays just before and after the eclipse of the X-ray source by the companion star. In this case the modulation of the UV emission should be clearly observed but in some cases can also be strongly suppressed because of the obscuration of the hottest parts on the stellar surface by the large accretion disk.

The $\gamma$-ray emission features from LMXBs can differ from those observed in HMXBs. The differences are caused in general by the optical depths for $\gamma$-rays in the radiation field of irradiated stars in LMXBs being lower than those for HMXBs. We note that a specific binary system may differ significantly from the simplified picture discussed in this paper. The main geometrical complications can be introduced by the inhomogeneous irradiation of the star by an X-ray source (anisotropic X-ray source) and the elliptic orbit of the X-ray source around the companion star. Moreover, our analysis is based on the assumption that the possible cascade initiated by primary electrons and $\gamma$-rays develop mono-directionally through the binary system. The structure of the magnetic field of the star can influence the paths of secondary cascade $e^{ \pm}$pairs. As a result, the escaping secondary $\gamma$-rays can form complicated structures on the sky (see Sierpowska \& Bednarek 2005).

We may ask whether absorption of $\gamma$-rays should not occur efficiently in the radiation field of the X-ray source on the surface of the accreting neutron star. We show below that this is not the case provided that $\gamma$-rays are injected at some reasonable distance from the X-ray source. Assuming the characteristic dimension of the X-ray source equal to $\sim 10^{5} \mathrm{~cm}$ (the order of the polar cap on the neutron star surface) and the luminosity of the $\mathrm{X}$-ray source $10^{38} \mathrm{erg} \mathrm{s}^{-1}$, we estimate the characteristic temperature of the X-ray source on $T_{\mathrm{X}} \approx 6 \times 10^{7} L_{38}^{1 / 4} R_{5}^{-1 / 2} \mathrm{~K}$, where $R_{\mathrm{X}}=10^{5} R_{5} \mathrm{~cm}$ is the characteristic dimension of the X-ray source. For simplicity, we note that we assumed the black-body type emission from the X-ray source. From this X-ray source, we estimate the distance, $D$, at which the optical depths in the radiation of this $\mathrm{X}$-ray source become lower than unity from $\tau=D \sigma_{\gamma \gamma \rightarrow e^{ \pm}} n_{\mathrm{x}}$, where $\sigma_{\gamma-\gamma \rightarrow e^{ \pm}}$is the cross section for $e^{ \pm}$pair creation in $\gamma-\gamma$ collision and $n_{\mathrm{x}} \approx 20 T^{3}\left(R_{x} / D\right)^{2} \mathrm{~cm}^{-3}$ is density of X-ray photons at the distance $D$. By assuming that the cross section for the $\gamma-\gamma$ collision is inversely proportional to the energy of a $\gamma$-ray photon, we estimate that the optical depth becomes lower than unity for distances larger than $D \sim 10^{8} \mathrm{~cm}$ and energies of $\gamma$-rays above $\sim 10 \mathrm{GeV}$. We also note that most of the $\gamma$-rays move in the outward direction in respect to the location of the X-ray source. Thus, the geometrical effects can also decrease the optical depths in the X-rays. This estimate of the possible location of the $\gamma$-ray source is clearly consistent with the dimensions of the binary system.

Based on similar estimate, it is possible to show that the radiation field from the inner part of the accretion disk around the neutron star may not prevent the escape of $\gamma$-ray photons, even if they are created within or close to this region. For example, in the case of Her X-1 the inner disk temperature is estimated to be in the range $\sim\left(1.8-2.5 \times 10^{4}\right) \mathrm{K}$ (Sazonov 2009) and the disk inner radius is at a distance of a few $\sim 10^{8} \mathrm{~cm}$ from the neutron star (Ghosh \& Lamb 1979). We estimate the mean free path for $\gamma$-rays propagating in this radiation to be $\sim 5 \times 10^{9} \mathrm{~cm}$. This is clearly larger than the dimension of the disk inner radius. Therefore, $\gamma$-rays can escape from the inner part of the accretion disk without significant absorption. We note that the region of particle acceleration may be located at some distance from the hot neutron star surface and the inner accretion disk but still inside the binary systems. For example, there are models that propose the acceleration of particles within the jets that are observed in the case of some LMXBs (e.g. Sco X-1).

We can envisage a realistic situation in which the absorption of $\mathrm{TeV} \gamma$-rays in the radiation of the X-ray source can be safely neglected. For example, in the case of Her X-1, the X-ray source can be produced on the neutron star surface by the accretion of matter. However, the acceleration of electrons (and production of the primary $\gamma$-rays) can occur close to or within a very turbulent, magnetized region at the inner accretion disk radius where the pressure of the accreting matter is balanced by a strongly magnetized rotating neutron star magnetosphere (the surface magnetic field of Her X-1 is estimated to be $2.9 \times 10^{12} \mathrm{G}$, e.g., Karino 2007). The inner radius of the accretion disk in Her $X-1$ is then expected to be at a distance of a few $10^{8} \mathrm{~cm}$ from the neutron star. In the case of Sco X-1, the production of $\gamma$-rays can occur inside part of the jet at some distance from the neutron star and the inner disk, but still inside the binary system.

Acknowledgements. This work is supported by the Polish MNiSzW grant No. N203 390834.

\section{References}

Aharonian, F., Akhperjanian, A. G., Aye, K.-M., et al. 2005a, Science, 309, 746 Aharonian, F., Beilicke, M., Aharonian, F., et al. 2005b, A\&A, 439, 635 Albert, J., Aliu, E., Anderhub, H., et al. 2006, Science, 312, 1771

Bednarek, W. 2000, A\&A, 363, 646

Bednarek, W. 2006a, MNRAS, 368, 579 
W. Bednarek and J. Pabich: Optical depths for gamma-rays in LMXBs

Bednarek, W. 2006b, MNRAS, 371, 1737

Bednarek, W. 2007, A\&A, 464, 259

Bednarek, W. 2009a, A\&A, 495, 919

Bednarek, W. 2009b, MNRAS, 397, 1420

Böttcher, M., \& Dermer, C. D. 2005, ApJ, 634, 81

Brazier, K. T. S., Carraminana, A., Chadwick, P. M., et al. 1990, A\&A, 232, 383

Dubus, G. 2006, A\&A, 451, 9

Fomalont, E. B., Geldzahler, B. J., \& Bradshaw, C. F. 2001, ApJ, 512, L121

Georganopoulos, M., Aharonian, F. A., \& Kirk, J. G. 2002, A\&A, 388, L25

Gorham, P. W., Cawley, M. F., Fegan, D. J., et al. 1986, ApJ, 308, L11

Ghosh, P., \& Lamb, F. 1979, ApJ, 234, 296

Gottlieb, E. W., Wright, E. L., \& Lillier, W. 1975, ApJ, 195, L33

Harding, A. K., \& Gaisser, T. K. 1990, ApJ, 358, 561

Hasinger, G., \& van der Klis, M. 1989, A\&A, 225, 79

Karino, S. 2007, PASJ, 59, 961

Kirk, J. G., Ball, L., \& Skjaeraasen, O. 1999, APh, 10, 31

Lamb, R. C., Cawley, M. F., Fegan, D. J., et al. 1988, ApJ, 328, L13

Levinson, A., \& Blandford, R. 1996, ApJ, 456, L29
Liu, Q. Z., van Paradijs, J., van den Heuvel, E. P., et al. 2007, A\&A, 469, 807

Maraschi, L., \& Treves, A. 1981, MNRAS, 194, 1

Milgrom, M. 1976, ApJ, 208, 191

Moskalenko, I. V., Karakuła, S., \& Tkaczyk, W. 1993, MNRAS, 260, 681

Nagase, F. 1989, PASJ, 41, 1

Reynolds, P. T., Cawley, M. F., Fegan, D. J., et al. 1991, ApJ, 382, 640

Romero, G. E., Kaufman Bernado, M. M., \& Mirabel, F. 2002, A\&A, 393, 61

Sierpowska, A., \& Bednarek, W. 2005, MNRAS, 356, 711

Sierpowska-Bartosik, A., \& Torres, D. 2007, ApJ, 671, 145

Sierpowska-Bartosik, A., \& Torres, D. 2009, ApJ, 693, 1462

Tavani, M., \& Arons, J. 1997, ApJ, 477, 439

Tonwar, S. C., Gopalakrishnan, N. V., Gupta, S. K., et al. 1991, PRL, 67, 2248

Sazonov, A. N. 2009 [arXiv:0912.0706]

Steeghys, D., \& Casares, J. 2002, ApJ, 568, 273

Vestrand, W. T., \& Eichler, D. 1982, ApJ, 261, 251

Vrtilek, S. D., Penninx, W., Raymond, J. C., et al. 1991, ApJ, 376, 278

White, N. E., Swank, J. H., \& Holt, S. S. 1983, ApJ, 270, 711 